Revue d'histoire de l'Amérique française

RAS REVUE D.HISTOIRE DE L'AMÉRIQUE FRANÇAISE

\title{
Saint Antoine Daniel, martyr canadien (suite et fin)
}

\section{Fernand Potvin}

Volume 10, numéro 3, décembre 1956

URI : https://id.erudit.org/iderudit/301775ar

DOI : https://doi.org/10.7202/301775ar

Aller au sommaire du numéro

Éditeur(s)

Institut d'histoire de l'Amérique française

ISSN

0035-2357 (imprimé)

1492-1383 (numérique)

Découvrir la revue

Citer cet article

Potvin, F. (1956). Saint Antoine Daniel, martyr canadien (suite et fin). Revue

d'histoire de l'Amérique française, 10(3), 391-415.

https://doi.org/10.7202/301775ar d'utilisation que vous pouvez consulter en ligne.

https://apropos.erudit.org/fr/usagers/politique-dutilisation/ 


\title{
SAINT ANTOINE DANIEL, MARTYR CANADIEN * \\ (Suite et fin)
}

\begin{abstract}
Ainsi, une mort glorieuse avait été réservée au Père Daniel afin que fût mise en lumière sa vie obscure, tout entière passée au service du Seigneur: "Son zele l'a accompagné iusqu'à la mort, qui ne l'a pas surpris au dépourueu,quoy qu'elle ait esté bien subite. Car il portoit tousiours son ame entre ses mains, y ayant plus de neuf ans qu'il demeuroit dans les places les plus frontieres de ce pays, et dans les Missions les plus exposées à l'ennemy, attendant auec esperance et amour le bonheur de la mort, qui luy est écheuë en partage $\gg .^{24}$ Mais cette mort était plus qu'une mort, elle était un martyre. Martyr de la charité et du zèle apostolique, le Père Daniel l'a été sans aucun doute possible: «Fuyez, mes Freres, avait dit le Pere à ses nouveaux Chrestiens, et portez auec vous vostre foy iusqu'au dernier souspir. Pour moy, adiousta-t-il, ie dois mourir icy, tandis que i'y verray quelque ame à gagner pour le Ciel; et y mourant pour vous sauuer, ma vie ne m'est plus rien, nous nous reuerrons dans le Ciel $\gg{ }^{25}$ Comme l'avait fait son Maître, «le Père Daniel est mort en imitant le Bon Pasteur qui donne sa vie pour ses brebis, tandis que le mercenaire prend la fuite $\gg .{ }^{26}$

Martyr de la foi, Antoine Daniel l'a été aussi : ce fait, beaucoup plus difficile à prouver, a cependant été reconnu véridique par un jugement officiel de l'Église. Aussi, sans reprendre les témoignages apportés à cette fin au procès de canonisation, nous contenterons-nous de rappeler brièvement les principales circonstances de la mort du Père Daniel qui font de lui un martyr de la foi.
\end{abstract}

* Voir notre Revue, vol. VIII (3) : 395-414; (4) : 556-564; IX (1) : 74$92 ;(2): 236-250 ;(3): 392-409 ;(4): 562-570 ; \mathrm{X}(1): 77-92 ;(2): 250-256$. 24 P. Ragueneau, Q $1649,4 \mathrm{~d}$.

25 Ibid., $4 \mathrm{~d}$.

26 Bressani, 182. 
A l'arrivée des Iroquois, le Père Daniel achevait sa messe. Mère de l'Incarnation rapporte: "Il était encore dans ses habits sacerdotaux lorsqu'il entendit le tumulte des ennemis et sans se donner le loisir de quitter son aube, il court de cabane en cabane... ».27 Quand les Iroquois se dirigèrent vers la chapelle pour y massacrer les chrétiens qui s'y trouvaient réunis, le Père Daniel sortit seul à leur rencontre: «Alors ce saint homme, avec un port tout plein de majesté, aborda l'ennemi, qui en fut tout effrayé: il leur parle de Dieu, leur prêche hautement la foi, et leur reproche leur trahison $\gg .^{28}$ Revenus de leur surprise, les Iroquois le couvrirent de flèches tandis qu'un coup d'arquebuse, tiré en pleine poitrine mettait fin à sa vie: "Ce fut alors que ces Barbares se ruerent sur luy, auec autant de rage que si luy seul eust esté l'objet de leur haine. Ils le dépoüillent nud, ils exercent sur luy mille indignitez, et il n'y en eût quasi aucun qui ne voulust prendre la gloire de luy auoir donné son coup, mesme le voyant mort $\gg .^{29}$

D'après plusieurs témoins, ${ }^{30}$ ces actes de violence inouie qui suivirent la mort du Père Daniel ne peuvent s'expliquer autrement que par la haine des Iroquois contre son caractère de prêtre de Dieu que ses paroles et ses vêtements sacerdotaux leur avaient clairement fait reconnaître. Les Iroquois, à l'instigation des Hollandais surtout, ${ }^{31}$ redoutaient ces «sorciers» dont la nouvelle religion, si opposée à leurs mœurs immorales, serait funeste à leur nation. D'après eux, les malheurs et les catastrophes survenus à leurs ennemis, les Hurons, ne pouvaient avoir d'autres causes.

Le sang versé est un témoignage qui ne peut pas mentir. Aussi, selon le Père Bressani, plusieurs sauvages, même infidè-

27 Marie de l'Incarnation, 4: 228.

28 Ibid., $4: 229$.

29 P. Ragueneau, Q 1649 , 4g.

30 Cardinale Antonio Vico, éd., Quebecen. Beatificationis seu Declarationis Martyrii Servorum Dei Joannis de Brébeuf ...., Positio super Introductione Causae, XIV testis, Sœur M. S. Lachance, 528; XVIII testis, A. E. Jones, 577-8.

31 Bressani, 180-1; voir aussi P. Ragueneau au R.P. Cl. de Lingendes, [1648], dans L. Campeau, "Protomartyr de la Huronie », 162. 
les, furent convaincus de la vérité de la foi chrétienne par la mort héroïque de leur pasteur :

La mort du Père Antoine fut précieuse, nonseulement devant Dieu, mais aussi devant les hommes. Les Sauvages, même infidèles, admiraient une force d'âme si extraordinaire, et un si grand mépris de la vie. Sa mort fut une prédication éloquente, et gagna plus de cœurs à Dieu que n'avaient pu faire bien des années de sa vie. Elle servit de motif de crédibilité à plusieurs obstinés, qui avaient jusque là refusé de croire, et qui en conclurent la vérité de nos mystères. "Ce n'était pas pour lui-même, » disaientils, «mais pour nous, que le bon Arontoin (c'est ainsi qu'ils l'appelaient) a voulu exposer sa vie et mourir. La foi qui est capable d'inspirer de pareils sacrifices, doit être sainte, et des hommes, qui nous aiment jusqu'à mourir pour nous, ne veulent certainement ni nous tromper, ni nous perdre. 》

D'autres Sauvages, qui avaient d'abord tourné notre sainte religion en ridicule, dirent aussi : « $L a$ foi est évidemment une chose importante, puisque ces Européens exposent si volontiers leur vie, afin de l'établir et de la conserver, et il faut qu'ils soient bien persuadés de ce qu'ils nous prêchent, et en particulier de l'existence d'une vie bienheureuse, puisqu'ils font si courageusement le sacrifice de la vie présente. » Un grand nombre d'infidèles, touchés par ces motifs, vinrent nous demander le baptême $\gg^{32}$

Témoins de son inlassable dévouement, ils reconnurent donc dans la mort du Père Daniel le suprême acte de foi en ce Dieu qu'il leur avait annoncé sans relâche. Le Père Daniel mourut vraiment en haine de la foi puisque sa mort, pour plusieurs sauvages, fut la preuve décisive en faveur de notre foi..$^{33}$

La grâce du martyre demeure toujours un don gratuit que Dieu, dans sa souveraine liberté, accorde à ceux que son amour a choisis. Comme toutes les grâces, cependant, celle-ci doit trouver

32 Bressani, 184-5; c'est nous qui soulignons.

33 Cardinale Antonio Vico, éd., Quebecen. Beatificationis..., Positio super Introductione Causae, X testis, A. Melançon, S.J., 505-6; XII testis, P. A. Fournet, P.S.S., 516-7. 
dans l'âme privilégiée des dispositions qui attirent sur elle, en quelque sorte, les bénédictions de Dieu. Si la grâce du martyre est un don, elle est aussi, du moins dans une certaine mesure, une récompense.

Le martyre du Père Daniel nous permet de découvrir sa vie antérieure sous un jour nouveau, il aide à comprendre la valeur inappréciable de quatorze années d'apostolat missionnaire, vécues presque sans interruption au milieu d'un peuple à peine civilisé. A la lumière du martyre, nous essayerons de percer le voile qui dérobe à nos yeux la vie intérieure de saint Antoine Daniel.

\section{Chapitre VIII}

\section{ITINERAIRE SPIRITUEL}

LIMINAIRE - LES GRANDES ÉTAPES DE SA VIE - DIVERS TÉMOIGNAGES: Le JeUnE, LALlemant, BRESSANI, RAGUENEAU MARIE DE L'INCARNATION ET L'ESPRIT DU SACRÉ VERBE INCARNÉ - DeUX aPPARITIONS AU PÈRe CHAUMONOT.

Pour reconstituer avec quelque exactitude la vie spirituelle de saint Antoine Daniel - ce qui déjà est une tâche extrêmement difficile et délicate - avouons d'abord qu'il nous manque les documents les plus essentiels, tels que seraient des notes ou un journal spirituel. Nous possédons, il est vrai, deux écrits ${ }^{1}$ dont l'authenticité - celle même des Relations - ne peut guère être mise en doute. Toutefois, ce ne sont pas là des lettres qui nous dévoilent sa vie intime, comme celles de Marie de l'Incarnation par exemple, mais une simple relation de faits divers, des lettres de nouvelles, sans plus. Même s'il est possible, par une lecture attentive, d'y saisir quelques données sur la personnalité du Père Daniel, ces lettres sont tout à fait impropres à nous renseigner sur sa vie spirituelle.

Une seule voie, reste donc ouverte: le témoignage de ses contemporains. Sa vie également, avec tout son cortège de cir-

1 a) Lettre du Père A. Daniel au P. Le Jeune, «Isle» des Algonquins, 7 juillet 1636, Q 1636, 69-70; texte et commentaire, voir notre Revue, IX (2): 242-243.

b) Extrait d'une lettre du Père A. Daniel au P. Le Jeune, Huronie, Juillet 1638, Q 1638, 26-27; texte et commentaire, voir notre Revue, IX (4) : 564-656. 
constances, porte un témoignage d'une exceptionnelle valeur sur l'homme parce qu'elle explicite peu à peu les desseins de la Providence à son égard. Nous rappellerons donc brièvement les principales étapes de la vie du Père Daniel, essayant cette fois d'y découvrir les intentions qui les vivifient et le potentiel spirituel qu'elles supposent, toujours cependant à la lumière du sommet ultime où cette âme est parvenue, la sainteté du martyre. Ensuite, nous essaierons de dégager les quelques constantes parmi les observations que nous avons pu relever chez des témoins aussi autorisés que les Pères Le Jeune, Lallemant, Bressani, Ragueneau; en particulier, nous nous arrêterons à un texte capital de Marie de l'Incarnation où elle décrit les sublimes effets de l'Esprit du sacré Verbe Incarné, un don mystique qu'elle affirme avoir été accordé au Père Daniel. Enfin, il ne faut pas oublier qu'après sa mort, le saint apparut à deux reprises au Père Pierre Chaumonot. Ces apparitions, attestées sous la foi du serment, revêtent un caractère spécial d'authenticité; elles nous laissent entrevoir surtout le foyer intime de sa vie spirituelle d'où sont partis les élans généreux qui l'ont peu à peu acheminé à la sainteté et au martyre.

Fils d'une excellente famille, ${ }^{2}$ le jeune Antoine Daniel entra dans la Compagnie de Jésus après avoir achevé, disent les catalogues de la Province de France, ${ }^{3}$ deux années de philosophie et une année de jurisprudence. Visiblement, il se dirigeait, à l'exemple de son frère Adrien peut-être, ${ }^{4}$ vers la profession d'avocat. Quel événement modifia donc soudainement cette orientation et lui fit tourner ses regards vers la Compagnie de Jésus ? Sa vocation avait-elle rencontré de sérieuses oppositions de la part de ses parents ou de sa famille? A notre avis, le changement dut se produire plutôt chez le jeune Antoine lui-même: appel plus

2 «Il estoit natif de Diepe, de parents tres honnestes et tres gents de bien », A. Melançon, éd., Mémoires touchant la Mort \& Les Vertus des Pères Isaac Jogues Anne de Noue, Anthoine Daniel Jean de Brebeuf Gabriel Lallement, charles garnier Noel Chabanel \& Un Seculier René Goupil (Montréal, s.d.), 109; à l'avenir: Mémoires.

3 Voir notre Revue, VIII (3): 409.

4 Ibid. 
impérieux, soumission tardive à la grâce ? Quoi qu'il en soit, nous retrouvons là un véritable signe de prédilection, assez fréquent, il est vrai, mais qui déjà nous révèle une âme d'une remarquable docilité à la voix intérieure.

Le Père Daniel eut le privilège de faire sa seconde année de noviciat sous la direction du Père Louis Lallemant, le grand maître français de la spiritualité ignatienne au début du dixseptième siècle; il le retrouva un peu plus tard au collège de Clermont comme professeur de théologie. ${ }^{5}$ Est-il possible d'apprécier l'influence que le Père Lallemant exerça sur son jeune disciple ? Ici plus qu'ailleurs, nous en sommes réduits à de simples hypothèses, car aucun document n'y fait même allusion. Il est bon de rappeler cependant que la seconde année de noviciat est une étape décisive dans la formation du jésuite puisqu'elle le conduit aux vœux perpétuels et que les années subséquentes, consacrées avant tout aux études, se nourrissent des convictions personnelles acquises auparavant. De plus, le Père Daniel, comme plusieurs de ses confrères, ${ }^{6}$ ne fit pas de troisième année de probation: autre raison qui nous incite à croire que cette année de noviciat, accomplie sous la direction d'un maître aussi réputé que le Père Lallemant, fut une étape importante dans sa vie spirituelle.

$\mathrm{Au}$ sujet de la doctrine spirituelle du célèbre Jésuite, le Père Latourelle écrit: "Cette doctrine n'est pas un produit insolite, différent de l'esprit de l'Évangile ou de celui des Exercices de saint Ignace. Ce qui la caractérise, c'est un accent mis plus fort sur certains points de la vie spirituelle, par exemple sur la pureté et la garde du cœur, le recueillement intérieur et l'union à Dieu, la docilité entière à la conduite de l'Esprit-Saint $\gg .^{7}$ Mais ce que nous connaissons de la spiritualité et des vertus du Père Daniel appartient trop, en effet, au fonds commun de l'Évangile et des Exercices de saint Ignace pour que nous puissions l'attribuer sans plus à une influence directe du Père Lallemant. Il semble bien que celui-ci marqua son disciple plutôt par l'exem-

5 Voir notre Revue, VIII (3) : 410.

6 Notamment le Père de Brébeuf, voir Latourelle, 2: 193.

7 Latourelle, 2 : 196. 
ple de sa vie que par tel ou tel point de sa «doctrine». Ainsi, nous savons que le Père Lallemant « demanda pendant trois ans d'être envoyé aux missions étrangères, et particulièrement en celle du Canada, quoiqu'on n'y fasse pas tant de conversions qu'ailleurs; mais elle est plus féconde en travaux et en croix; elle est moins éclatante, et contribue plus que les autres à la sanctification de ses missionnaires. C'est ce qui la lui faisait aimer par-dessus toutes les autres : et n'ayant pu obtenir d'y aller luimême, il s'employa toujours à procurer à cette mission de fervents ouvriers, et à lui rendre en France tous les services qui dépendaient de lui $\gg^{8} \mathrm{Si}$, dès son noviciat, le Père Daniel se sentit appelé aux missions de la Nouvelle-France, il dut trouver dans son Père Maître de puissants encouragements. De même, la patience et la douceur du Père Lallemant ont retenu l'attention de son biographe, le Père Champion: «La patience et la douceur sont les plus nobles effets et les plus solides preuves de la force. Le P. Lallemant a excellé en l'une et en l'autre. Il souffrait de si bonne grâce, qu'à le voir souffrir, on eût jugé qu'il n'eût rien souffert. Comme il était parfaitement maître de lui-même, jamais on ne remarquait en lui d'inégalité d'esprit, ni d'humeur. Il possédait son âme dans une paix profonde, et la sérénité paraissait toujours sur son visage $\gg .^{9}$ Ces vertus que le Père Daniel pratiqua à un degré si éminent, il en avait eu un exemple insigne dans la vie de son Père Maître, exemple plus fécond peutêtre que ses paroles. Aussi croyons-nous que le rayonnement de sainteté qui émanait de sa personne laissa une trace profonde dans la vie du Père Daniel, l'exemple demeurant toujours l'argument le plus décisif, le seul qui entraîne véritablement.

Nous ne pouvons pas non plus ignorer la présence du Père de Brébeuf, d'abord au collège de Rouen de 1623 à 1625 alors que le Père Daniel y commençait sa régence, ${ }^{10}$ et surtout au collège $d^{\prime} \mathrm{Eu}$ en 1631-32,11 année qui précéda immédiatement le

8 A. Pottier, éd., La Vie et la Doctrine spirituelle du Père Louis Lallemant de la Compagnie de Jésus (Paris, 1924), 22-23.

${ }^{9} \mathrm{~A}$. Pottier, éd., La Vie et la Doctrine spirituelle du Père Louis Lallemant de la Compagnie de Jésus, 22.

10 Voir notre Revue, VIII (3) : 410.

11 Ibid.: 412. 
départ du Père Daniel pour la Nouvelle-France. Dès cette époque, le journal spirituel de « l'apôtre au cœur mangé » est rempli de brûlants désirs d'immoler sa vie pour la salut des âmes. C'est en effet au mois de mai 1631 que le Père de Brébeuf signa de son sang la promesse de servir le Christ jusqu'à la mort. ${ }^{22}$ Cette ardente charité inspirait les conseils que le Père de Brébeuf, déjà riche de trois années d'apostolat en Huronie, devait prodiguer au futur missionnaire. Plus tard, Brébeuf sera encore à ses côtés, lui donnant chaque jour l'exemple d'une ténacité invincible jointe à une douce mansuétude. ${ }^{13}$ A Québec en 1633, en Huronie les deux années suivantes, puis de 1638 à 1641 et enfin de 1644 jusqu'à sa mort, le Père Daniel vivra en compagnie de cet apôtre incomparable. C'est dire déjà l'influence forte et profonde que le « géant des missions huronnes », mais aussi le mystique et le futur martyr, exerça sur lui.

En Nouvelle-France, le Père Daniel travailla l'espace de seize années. A la regarder dans son ensemble, cette dernière période de sa vie se partage nettement en deux étapes: d'abord, une série de déplacements successifs: un an au Cap-Breton, un an à Québec pour étudier la langue, deux ans d'apostolat en Huronie, deux ans à Québec pour diriger le Séminaire huron; ensuite, un long ensemble de dix années de mission dont rien d'extraordinaire, même pas la guerre iroquoise, ne paraît avoir interrompu le labeur quotidien.

Comme tous les véritables apôtres, le Père Daniel connaîtra la Croix: aucune de ces étapes ne le conduira à un franc succès. Au contraire, la plupart se termineront par un échec douloureux. $\mathrm{Au}$ Cap-Breton d'abord, l'éloignement des indigènes ne lui permit pas d'exercer un véritable apostolat missionnaire: c'était pourtant à cause de cet idéal qu'il avait quitté la France. A Québec, durant l'hiver de 1633-34, il en profita, certes, pour étudier la langue huronne sous la conduite du Père de Brébeuf, mais la première ambition de ces deux apôtres n'était-elle pas de pénétrer le plus tôt possible en Huronie ? Enfin, voici le Père Daniel en pleine activité missionnaire: deux ans passent, le Père de 
Brébeuf, à son grand regret, ${ }^{14}$ doit lui demander de retourner à Québec pour prendre soin du séminaire huron. Humblement, le Père Daniel quitte le champ de son apostolat au moment où le besoin d'ouvriers se fait plus vivement sentir, car les Hurons se ferment de plus en plus à la parole de l'Évangile: le combat décisif pour le triomphe de la foi va s'engager bientôt. Il accepte pourtant de retourner en arrière des lignes.

1636-1638: le Père Daniel, à Québec, consacre toutes ses énergies au bon fonctionnement du séminaire dont le Père Le Jeune attendait de si merveilleux résultats. Mais cette entreprise, née avant l'heure, était irrémédiablement vouée à l'échec. Les événements le firent voir sans délai. Après avoir réussi à convaincre six jeunes gens de le suivre à Québec, le Père Daniel entreprend courageusement leur éducation. Au bout de quelques semaines, l'un d'eux était retourné chez lui, deux autres étaient morts et, pour comble de malheur, le Père Daniel, à son tour, tombait dangereusement malade, conséquence d'un trop violent surmenage. C'était le bilan de la première année. En 1637, le plus jeune des élèves retourne en Huronie tandis que quatre nouvelles recrues viennent compléter le nombre de six séminaristes, chiffre maximum pour les ressources de la Mission. Cette fois, la situation tourne au désastre: l'un des quatre nouveaux quitte bientôt les Pères suivi de près par les trois autres qui s'enfuient après avoir pillé le séminaire. Enfin, au printemps de 1638, le Père Daniel retourne en Huronie, accompagné des deux seuls séminaristes persévérants. A Québec, le séminaire huron retournait à l'état de projet, n'ayant plus ni maître ni écoliers pour en maintenir l'existence.

Ce sont là des faits qui devaient peser lourdement sur le cœur du Père Daniel, car un missionnaire ne consacre pas deux années de sa vie à une entreprise sans ressentir une profonde amertume s'il en constate l'apparente inutilité. Une épreuve de ce genre se situe parfaitement parmi celles que la Providence réserve d'ordinaire à un apôtre authentique. Issue de l'action, elle le purifie mieux que ne le ferait aucune pénitence volontaire parce que celle-ci est rarement exempte de vanité personnelle.

14 Voir ibid., 1: 194, 2: 53, note 3. 
Enfin, le Père Daniel va, semble-t-il, jouir d'une stabilité relative: dix années employées à l'évangélisation des quelques bourgades situées à la frontière est de la Huronie. Après tant de faux départs, il connaîtra le lent travail de l'ensemencement de la vie chrétienne dans des cœurs souvent fermés et hostiles, l'attente inquiète de la première récolte, toujours à la merci de la fragilité humaine. Ce long cheminement, dont les vœux solennels marquent en 1640 une étape décisive, achève de dépouiller le Père Daniel de toute ambition terrestre. Son simple status d'« Operarius » qu'il conservera jusqu'à son martyre est l'un de ses plus beaux titres: celui de l'ouvrier qui persévère dans la vigne du Seigneur depuis la première heure du jour.

Voilà comment nous apparaît, vue dans son ensemble, la carrière humaine du Père Daniel, carrière, est-il besoin de le dire, qui ne présente rien de particulièrement brillant. Sa vie, au Canada surtout, n'est d'abord qu'une longue suite d'échecs dont, certes, il n'est pas toujours le seul responsable; puis viennent dix années d'apostolat obscur où il connaîtra la solitude et l'absence de toute consolation humaine. ${ }^{15}$ Bref, chacune des étapes de sa vie lui est une occasion d'un renoncement de plus en plus exigeant. Dans un tel contexte, la mort violente qui lui était réservée ne put prendre au dépourvu ${ }^{16}$ un homme dont la vie n'en avait été que le constant apprentissage.

Parmi les divers témoignages que ses contemporains nous ont laissés, il n'est sans doute pas très difficile de découvrir certaines caractéristiques. Mais celles-ci demeurent trop incohérentes et trop peu nombreuses pour nous permettre de recomposer les traits d'une personnalité. Aussi devrons-nous parfois rappeler l'une ou l'autre circonstance de sa vie où se sont manifestées avec plus de lumière les vertus que ses compagnons d'apostolat ont admirées en lui.

15 Voir notre Revue, IX (4): 562-573; X (1) : 77-92.

16 « Son zele l'a accompagné jusques à la mort, quj ne l'a pas surpris au despourveu, quoy qu'elle ait esté bien subite », Mémoires, 108. 
Sa conduite extérieure et l'ensemble de son comportement ont toujours inspiré une grande confiance à ceux qui l'ont connu. C'est en effet, "un homme remarquable, bien digne fils de la Compagnie de Jésus, humble, obéissant ${ }^{17}$ que salue en lui le Père Ragueneau, en annonçant la nouvelle de son martyre. D'ailleurs, son admission à la profession solennelle des quatre vœux ${ }^{18}$ laissait prévoir ce jugement: cette admission au degré de profès étant d'ordinaire le signe d'une entière satisfaction de la part des supérieurs.

Mais le Père Daniel se distinguait avant tout par «une patience insurmontable, une douceur inalterable et avec une charité quj scavoit tout excuser tout supporter et tout aimer $\gg{ }^{19}$ Dans une lettre adressée au très révérend Père Mutius Vitelleschi, en 1636, le Père de Brébeuf indiquait les principales qualités dont le missionnaire de la Huronie devait surtout faire preuve: «Entre autres qualités qui doivent briller chez un apôtre de cette mission, la douceur et la patience doivent tenir le premier rang. Jamais ce pays ne portera des fruits que dans la douceur et la patience; et ce n'est ni par la force ni par l'autorité qu'on peut espérer jamais le conquérir $\gg .^{20}$ Cette vertu, à la fois naturelle et acquise, inspirait à ses supérieurs une telle confiance que souvent ils lui demandèrent de prendre à lui seul le soin de plusieurs bourgades. ${ }^{21}$ Rappelons aussi la tentative du séminaire huron: le Père Daniel fut désigné sans doute parce qu'à cette époque, il était le seul, outre le Père de Brébeuf, à bien maîtriser la langue huronne. Mais son adresse à ménager la susceptibilité de ses élèves ne lui rendit pas un moindre service. ${ }^{22}$ Faisant clairement allusion au Père Daniel, leur instructeur, le Père Le Jeune écrivait: "Ce n'est pas qu'il ne faille vne grande dexterité à les conduire, vne douceur et vne patience tres-insigne: car de se seruir d'aigreur parmi ces Nations, c'est

17 P. Ragueneau au T.R.P. Cl. de Lingendes, [1648], L. Campeau, « Protomartyr de la Huronie 》, 166.

18 Voir notre Revue, X (1): 82.

19 Mémoires, 108, (P. Ragueneau).

20 J. de Brébeuf au T.R.P. M. Vitelleschi, [1636], Latourelle, 2: 55.

21 Voir notre Revue, X (1) : 89-90, 92.

22 Voir notre Revue, IX (3) : 398-99. 
les jetter dans la reuolte.$_{.} 3$ Envers tous, son attitude était si prévenante que le Père Ragueneau a pu dire de lui : «... il nous a laissé apres soy l'exemple de toutes ses vertus, et à tous les sauvages, mesmes infidelles, une affection sy tendre pour sa memoire que je puis dire en verité qu'il a ravy le cœur de tous ceux quj l'ont jamais cogneu $\gg .^{24}$

En plus de cette charité exquise par laquelle il savait se faire tout à tous, le Père Daniel, à l'exemple de ses compagnons, possédait à un degré éminent le zèle apostolique. Sur ce point, les témoignages ne nous laissent aucun doute: "Son obéissance... son zèle pour le salut des âmes étaient admirables, et ce qui est encore plus extraordinaire, il possédait avec un cœur très-ardent une douceur tout à fait remarquable, qui le rendait un instrument très-puissant pour la conversion de ces infidèles ». ${ }^{25}$ Cet homme, aux allures si discrètes et si suaves, était en même temps dévoré par la soif du royaume de Dieu : "Il sembloit n'estre né que pour le salut de ces peuples, et n'avoit point de desir plus violent que de mourir pour eux $\gg .^{26}$ Cette charité trouva, à la fin de sa vie, un couronnement digne de son ardeur: après sa dernière retraite, en 1648 , où, plus que jamais, il s'était enflammé «dans les desirs de respandre et son sang et sa vie pour le salut des ames... il ne voulut pas prendre mesme un jour de repos, se sentant appellé de dieu dans les travaux de sa Mission où il porta ce feu du Ciel, dont sans doubte son ame estoit plus embrasée que jamais son corps ne l'ait esté, quoy que sainctement consumé dans le milieu des flammes $\gg .{ }^{27}$ C'est là l'épreuve suprême qui révèle un véritable apôtre: «Le Pere Antoine Daniel est mort en imitant le Bon Pasteur, qui donne sa vie pour ses brebis, tandis que le mercenaire prend la fuite $\gg .{ }^{28}$

Tout entier au service de son prochain, le Père Daniel possédait aussi la charité qui unit à Dieu par la prière. Dans un domaine aussi réservé, connu le plus souvent du seul confesseur,

23 P. Le Jeune, Q 1637, 60g.

24 P. Ragueneau, Mémoires, 110.

25 Bressani, 185.

26 P. Ragueneau, Mémoires, 109.

27 Ibid., 109.

28 Bressani, 182. 
nous devrons davantage nous contenter simplement des affirmations de ses contemporains. Ainsi, en sa qualité de supérieur, le Père Ragueneau n'ignorait pas l'excellence des âmes qui lui étaient confiées: "C'était un homme remarquable... extrêmement familier avec Dieu par la pratique de l'oraison ». ${ }^{29}$ Cette union intime avec Dieu n'était pas d'ailleurs un fruit d'arrièresaison, car le Père René Ménard, missionnaire en Huronie depuis 1641, en avait déjà reçu la confidence. Ces aveux durent l'impressionner vivement puisque, quinze ans plus tard, il en rappelle encore le souvenir: "I'auois souuent ouy dire au Pere Daniel et au Pere Charles Garnier, lorsqu'ils estoient aux Hurons, que plus ils s'estoient veus delaissez et esloignez des consolations humaines, plus Dieu s'estoit emparé de leur cœur, et leur auoit fait sentir combien sa sainte grace l'emportoit par dessus toutes les douceurs imaginables qui se trouuent parmy les creatures $\gg^{30}$ Ce témoignage du Père René Ménard nous introduit directement aux splendeurs de l'Esprit du Sacré Verbe Incarné dont le Père Daniel avait été gratifié.

La «Thérèse du Nouveau Monde », Marie de l'Incarnation, nous a laissé, en effet, sur le Père Daniel et sur quatre de ses plus illustres compagnons, de Brébeuf, Jogues, Lallemant et Garnier, un témoignage d'une exceptionnelle valeur qu'il importe maintenant d'examiner avec attention. Dans une longue lettre, datée du 22 octobre 1649 et adressée à son fils, dom Claude Martin, elle décrit et explique la nature et les effets merveilleux de ce présent qu'elle nomme «l'Esprit du Sacré Verbe Incarné »:

Le présent le plus précieux en tout est l'esprit du sacré Verbe Incarné, quand il le donne d'une façon sublime, comme il le donne à quelques âmes que je connais de cette nouvelle Eglise, et comme il l'a donné à nos saints martyrs, les Révérends Pères de Brébeuf, Daniel, Jogues et Lalemant, qui ont fait paraître par leur généreux courage combien leur

29 P. Ragueneau au R.P. Cl. de Lingendes, [1648], L. Campeau, « Protomartyr de la Huronie », 166.

30 René Ménard, cité par le P. J. Lalemant, Q 1664, 6g. 
cœur était rempli de cet esprit et de l'amour de la croix de leur bon Maître. C'est cet esprit qui fait courir par mer et par terre les ouvriers de l'Evangile, et qui les fait des martyrs vivants avant que le fer et le feu les consume. Les travaux inconcevables qu'il leur faut endurer sont des miracles plus grands que de ressusciter les morts.

Pour venir au particulier, je vous dis que c'est un présent, parce qu'il ne s'acquiert pas dans une méditation. Il peut néanmoins arriver que Dieu le donne à une âme qui aura été fidèle en quelque occasion de conséquence pour sa gloire, et même en une petite, faite avec un parfait amour de Dieu et une entière haine de soi-même. Mais, pour l'ordinaire, il le donne après beaucoup de sueurs dans son service, et de fidélités à sa grâce. Ce don est une intelligence de l'esprit de l'Evangile et de ce qu'a dit, fait et souffert nostre adorable Seigneur et Maitre, avec un amour dans la volonté conforme à cette intelligence. Concevez un point de la vie cachée du Fils de Dieu, cela contient une sainteté que les plus hauts Séraphins adorent, et ils reconnaissent qu'ils ne sont que des atomes et des néants en comparaison des sublimes occupations intérieures de ce divin Sauveur. Considérez encore les trois années de sa conversation avec les hommes, ses entretiens particuliers, ses prédications, ses souffrances, sa passion, sa mort; vous direz que ces trois années ont porté ce qu'il y a de plus divin: il nous a donné ou acquis tous les biens de la grâce et de la gloire. Par la distinction des états de cet adorable Maître, nous connaissons la différence des nôtres avec quelque proportion, car, à Dieu ne plaise que nous fassions de la comparaison entre Lui et nous ! Dans cet aveu, la compagnie familière que l'on a avec Dieu surpasse ce que j'en ai dit ci-dessus, et donne une générosité d'une bien autre trempe que la première. Cet excellent Sermon de la Montagne: Bienheureux sont les pauvres d'esprit, etc., et celui de la Cène sont la force et le bastion des âmes à qui Dieu fait ce présent. Ne vous imaginez pas qu'en cette occupation il se passe rien dans l'imagination ou dans le corps; non, le tout est dans la substance de l'esprit par une infusion de grâce purement spirituelle. En cet état, on ne pratique pas seulement les maximes 
que vous savez, on se sent encore poussé à la pratique de toutes celles de l'Evangile qui sont conformes à l'état où nous sommes appelés, et aux emplois ou l'obéissance nous engage. L'âme fait plus de chemin en un jour dans cette disposition, qu'elle ne ferait en toute autre dans un mois. Cette approche amoureuse du sacré Verbe Incarné porte dans l'âme une onction qui ne se peut exprimer, et dans les actions une sincérité, droiture, franchise, simplicité, fuite de toutes obliquités; elle imprime dans le cœur l'amour de la croix et de ceux de qui l'on est persécuté; elle fait sentir et expérimenter l'effet des huit béatitudes d'une manière que Dieu sait et que je ne puis dire.

Tous ces heureux effets et beaucoup d'autres que je ne dis pas, viennent de l'onction et de l'attrait continuel avec lequel l'Esprit de Jésus emporte l'âme. Cet Esprit persuade, convainc et attire si doucement, qu'il n'est pas possible de lui rien refuser, et de plus, il agit dans l'âme comme dans une maison qui lui appartient entièrement. Cette douce persuasion est son langage, et la réponse de l'âme est de se laisser emporter en cédant amoureusement. Ce sont de mutuels regards et des intelligences si pures, que nos paroles sont trop basses pour les énoncer. L'âme, sans faire peine à la nature, qu'elle attire facilement après soi, se voit tranquille dans les choses les plus pénibles et difficiles. Quand même la nature, par faiblesse et infirmité, serait surprise par quelque tort ou injure qu'on lui fait, l'âme s'en aperçoit aussitôt, et la nature n'a plus de force; la paix et l'onction intérieure fait même qu'on aime ceux qui ont fait l'injure. Il en est de même de tout le reste. L'âme est humblement courageuse et sans respect humain dans les occasions où il y va de la justice et de l'équité, néanmoins avec une soumission entière de jugement à ceux qui la dirigent.

Dans cet état, l'âme ne commet plus d'indiscrétions, parce qu'elle est unie à Dieu d'une façon qui la rend libre; elle voit clair en toutes ses opérations, n'étant plus dans des transports de désir et d'amour comme elle a été autrefois. C'est ici la liberté des enfants de Dieu, qui les introduit dans sa familiarité sainte, par la confiance et par le libre accès qu'il lui donne. Dans les états passés, elle était dans un enivrement et transport qui la faisait oublier elle- 
même; mais ici elle est à son Bien-Aimé et son Bien-Aimé est à elle avec une communauté d'intérêts et de biens, si j'ose ainsi parler. Cela fait qu'elle s'expose à tout pour sa gloire, et que nonobstant toutes les croix qui se rencontrent, elle pratique suavement la loi du parfait anéantissement pour n'être plus, et afin qu'il soit tout et l'unique glorifié. Ce n'est pas qu'il se trouve des occasions où les croix se rendent plus sensibles, et qu'il ne s'y commette même des imperfections, mais cela passe vite; l'âme s'humilie et fait facilement sa paix par l'agrément de son humiliation. Car remarquez que plus l'âme s'approche de Dieu, plus elle connaît son néant, et quoiqu'elle soit élevée à un très haut degré d'amour, elle ne laisse pas de s'abaisser à un très profond degré d'humilité; ces deux dispositions s'accordent parfaitement ensemble, ce qui me fait connaître la vérité de cette parole de Notre-Seigneur, que celui qui s'humilie sera élevé. ${ }^{31}$

Il est intéressant de constater que Marie de l'Incarnation, n'ayant jamais connu personnellement le Père Daniel, ${ }^{32}$ ait pu discerner en lui, avec autant de certitude, ce don si extraordinaire. Mais, plutôt qu'une révélation surnaturelle, sa vie héroïque, soutenue sans défaillance pendant si longtemps, lui a suffi pour reconnaître la présence de ce don précieux: «... les Révérends Pères de Brébeuf, Daniel, Jogues et Lallemant, ${ }^{33}$... ont fait paraître par leur généreux courage combien leur cœur était rempli de cet esprit et de l'amour de la croix de leur bon Maître. C'est cet esprit qui fait courir par mer et par terre les ouvriers de l'Évangile ... $\gg^{34}$

Cet «esprit du sacré Verbe Incarné » se présente d'abord comme un don mystique parce que purement gratuit, mais dont

31 Marie de l'Incarnation, 4: 255-262; outre les annotations indispensables de l'éditeur, dom A. Jamet, voir le commentaire du P. Latourelle dans Etude sur les écrits de saint Jean de Brébeuf, 2: 185-191 ainsi que la courte présentation du P. Rouquette dans Textes des Martyrs de la NouvelleFrance (Paris, 1947), 58-59.

32 Marie de l'Incarnation, $4: 228$, note 3.

33 Si Marie de l'Incarnation ne mentionne pas aussi les PP. Garnier et Chabanel, c'est probablement parce qu'ils n'ont pas encore été martyrisés au moment où elle écrit cette lettre.

34 Marie de l'Incarnation, 4: 255. 
l'effet primordial consiste dans un accroissement du zèle apostolique et de sa fécondité. ${ }^{35}$ Il est d'une qualité si sublime que l'âme, même très sainte, met habituellement beaucoup de temps à s'en rendre digne. "Ce don, dit Marie de l'Incarnation, est une intelligence de l'esprit de l'Évangile et de ce qu'a dit, fait et souffert notre adorable Seigneur et Maître, avec un amour dans la volonté conforme à cette intelligence $»^{36}$ Il s'agit donc à la fois d'une grâce de lumière et d'une grâce de force. La première fait pénétrer l'âme si avant dans l'esprit de Jésus qu'elle découvre spontanément la conduite, la décision qui seraient les plus conformes à ce divin Modèle, car « cet Esprit persuade, convainc et attire si doucement, qu'il n'est pas possible de lui rien refuser, et de plus, il agit dans l'âme comme dans une maison qui lui appartient entièrement ».37 La seconde verse dans l'âme une énergie nouvelle qui l'entraîne presque irrésistiblement à accomplir la souveraine volonté de Dieu. La chair est si pleinement assumée par l'esprit que rien n'offre plus d'obstacle aux inspirations de la grâce: "L'âme, sans faire peine à la nature, qu'elle attire facilement après soi, se voit tranquille dans les choses les plus pénibles et difficiles $\gg .38$

Le point peut-être le plus important à souligner, c'est la grande liberté, ou mieux encore, la libération que cet esprit réalise dans l'âme. L'Esprit du Sacré Verbe Incarné est, en effet, un don «supérieur à tous les états d'union, si élevés fussent-ils, qui pourraient exister sans lui. Il se situe au delà des extases et des ravissements. C'est « le plus haut point de la vie spirituelle et la consommation des Saints que de n'avoir plus de vie que dans le Christ, selon le mot de saint Paul ».39 Auparavant, l'âme, en pleine crise de croissance spirituelle, subissait les transports et les ravissements de l'extase; ${ }^{40}$ arrivée maintenant à un état plus stable, elle est entrée dans le rayonnement de la vie divine qui lui laisse une pleine liberté d'action: «Ne vous imaginez pas

35 R. Rouquette, éd., Textes des Martyrs ..., 59.

36 Marie de l'Incarnation, $4: 256$.

37 Ibid., 4: 259 .

38 Marie de l'Incarnation, 4: 259.

39 Latourelle, 2: 189.

40 Marie de l'Incarnation, 4: 260. 
qu'en cette occupation il se passe rien dans l'imagination ou dans le corps; non, le tout est dans la substance de l'esprit par une infusion de grâce purement spirituelle ».41 Plus loin, elle ajoute: "C'est ici la liberté des enfants de Dieu, qui les introduit dans sa familiarité sainte, par la confiance et par le libre accès qu'il lui donne. Dans les états passés, elle était dans un enivrement et transport qui la faisait oublier elle-même; mais ici elle est à son Bien-Aimé et son Bien-Aimé est à elle avec une communauté d'intérêts et de biens, si j'ose ainsi parler $\$^{42}$ Cette dernière phrase laisse entrevoir la familiarité extraordinaire à laquelle l'âme est parvenue dans ses relations avec Dieu. Nous voyons en même temps que ce don mystique est tout orienté vers l'action, ${ }^{43}$ parce qu'il affranchit totalement la volonté des aveuglements et des faiblesses inhérentes à la nature. L'esprit du Verbe Incarné respecte la liberté de l'âme à ce point qu'il n'atténue en rien les contre-coups douloureux que l'action lui fait subir: «Ce n'est pas qu'il se trouve des occasions où les croix se rendent plus sensibles, et qu'il ne s'y commette même des imperfections, mais cela passe vite; l'âme s'humilie et fait facilement sa

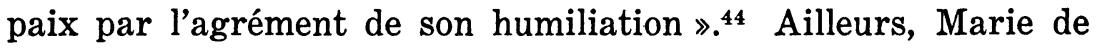
l'Incarnation insiste encore davantage sur ce fait que l'esprit du Verbe Incarné n'enlève rien aux souffrances de l'apostolat mais donne simplement la force de les accepter: "Cet esprit saint, cette union, dis-je, dont je vous parle, n'est pas celle de la gloire, elle en est seulement un avant-goût. Et ne pensez pas qu'elle rende toujours les travaux faciles, puisqu'elle ne redonde pas toujours dans les sens. Mais elle donne dans le fond de l'âme une force invincible pour les supporter, quelque pesants et pénibles qu'ils soient $\gg^{45}$

Ces perspectives rendent encore plus vraisemblables, croyons-nous, les traits de similitude avec Jésus-Christ que certaines circonstances de son martyre peuvent suggérer. ${ }^{46}$ Elles

41 Ibid., $4: 258$.

42 Marie de l'Incarnation, $4:$ 260-1.

43 Voir Latourelle, 2: 187.

44 Marie de l'Incarnation, 4 : 261-2.

45 Ibid., 4 : 292.

46 Voir notre Revue, X (2) : 254-255. 
expliquent surtout les conversions étonnantes que ses paroles ont produites: «...et comme s'il eust veu le paradis ouvert pour les Chrestiens, et l'enfer sur le point d'abismer tous les Infidelles, il leur parle d'un ton animé de l'esprit quj le possedoit; quj ayant faict bresche dans les cœurs quj jusques alors avoient esté les plus rebelles, il leur donna un cœur chrétien. Le nombre s'en trouve sy grand ... ».47 Ces mots: «il leur parle d'un ton animé de l'esprit quj le possedoit » pourraient sans doute s'interpréter selon leur sens usuel, mais après le témoignage de Marie de l'Incarnation sur le Père Daniel, ne serait-il pas plus exact d'y reconnaître les effets des grâces mystiques qu'il avait reçues ?

Dieu, d'ailleurs, ne tarda pas à manifester aux hommes la gloire dont il avait voulu récompenser son fidèle serviteur. Quelques jours après son martyre, le Père Daniel apparut en songe au Père Joseph-Marie Chaumonot, ${ }^{48}$ son ancien compagnon de mission; ${ }^{49}$ plus tard encore, la même apparition se renouvela une seconde fois. A la demande du Père Ragueneau, alors supérieur de la Mission canadienne, le Père Joseph Poncet, un vieil ami du Père Chaumonot, ${ }^{50}$ l'interrogea minutieusement sur ces visions. En même temps, il rédigea un nouveau récit ${ }^{51}$ des

47 P. Ragueneau, Mémoires, 105.

48 Des trois documents écrits par le Père Ragueneau, seule la lettre adressée au T.R.P. V. Caraffa indique le nom du Père Chaumonot, voir Rochemonteix, 2: 462; dans les Mémoires de 1652, il est également omis dans le texte mais le Père Ragueneau a inséré la note suivante qui révèle l'identité du Père N...: « Ce bon pere s'apparut apres sa mort à un (1) des nostres par deux diverses foys... (1) là fuit Pater Josephus Maria Chaumonot cui in somnis haec visa sunt. Paulus Ragueneau », Mémoires, 110.

49 Voir notre Revue, X (1) : 84 ; le P. Félix Martin explique ainsi pourquoi le Père Chaumonot fut gratifié de ces deux apparitions: "La mort du Père Daniel affecta vivement le Père Chaumonot. Ils avaient souvent travaillé ensemble; et une commune ardeur pour le bien unissait intimement leurs cœurs. Cette douce et sainte amitié mérita sans doute au P. Chaumonot la faveur de deux apparitions du généreux martyr », F. Martin, éd., Autobiographie du Père Chaumonot de la Compagnie de Jésus et son Complément, 91.

50 Voir ibid., 34-40.

51 Le $P$. Ragueneau avait déjà composé un récit des apparitions pour chacun des trois documents dont nous avons parlé plus haut, (voir notre Revue, X $(2): 250)$. En 1652, l'archevêque de Rouen demanda qu'on recueillît des témoignages pouvant servir à la canonisation des martyrs 
apparitions, rigoureusement conforme aux souvenirs du Père Chaumonot. Les soulignés indiquent les passages pour lesquels on trouvera de notables variantes en note:

Le Pere N., quelques jours après la mort du $P$. Anthoine Daniel estant en sa Mission au village de la conception aux Hurons, se mit à sommeiller après midy, pour se soulager de la lassitude de quelque travail extraordinaire du matin, à l'instruction des sauvages; et dans ce repos, il se vit en songe en l'assemblée de tous nos peres telle que nous faison deux ou trois foys l'année, pour conferer par ensemble des moyens d'avancer la conversion des sauvages. ${ }^{52}$ Là il vit parmy les autres le pere Daniel, avec la pensée qu'il estoit mort, mais là present miraculeusement. Il sentit alors un grand desir de luy parler; mais n'osoit aller à luy pour le respect de la compagnie et de l'attention que chacun portoit à ce quj

canadiens. Le Père Ragueneau, à la fin du premier chapitre de sa Relation de 1649 , apposa une déclaration officielle de son authenticité, Mémoires, III. Ensuite vient un second récit des apparitions au Père Chaumonot que confirment deux autres serments:

« Ego infrascriptus presbyter Societatis Jesu ad id a R.P. Paulo Ragueneau hujus missionis Sup.re delegatus, suprascriptum Patrem N, (quj me rogavit ne nomen suum declararem) accurate percunctatus sum de somnio seu visione supradicta, num ita se haberet, quam ille eo plane modo, uti jacet, se habuisse confirmavit. Eum autem, coram Deo judico fide dignum, talemque cui talis meritò divinitus conferantur. Quae duo videlicet narrationis praedictae et opinionis meae sinceritatem juratus affirmo. Kebeci 1652, 24 Aug. 1652. Joseph Poncet. Jhs.

Ego infrascriptus, jussus a R. P. Paulo Ragueneau, superiore meo, haec ut attestari non recusarem, iisque nomen meum subscribere, si vera sint: juratus affirmo versa esse », Mémoires, 112.

D'après ce passage: "Quae duo videlicet narrationis praedictae et opinionis meae sinceritatem juratus affirmo », il semble bien que ce second récit doive être attribué au Père J. Poncet et non au Père Ragueneau, contrairement à la notice bibliographique du Père L. Campeau que nous avons citée en appendice, page 155. Le second serment, non signé, proviendrait, soit d'un Père quelconque, soit, plus probablement, du Père Chaumont luimême comme le donnent à entendre ces mots: « haec ut attestari non recusarem, iisque nomen meum subaribere, si vera sint ».

52 « Une autre fois il fut veu assister à une assemblée que nous tenions touchant les moyens d'avancer la foy en ces pays; et alors il paroissoit nous fortifiant de son courage; nous remplissant de ses lumieres, et de l'esprit de dieu dont il estoit tout investy », P. Ragueneau, Mémoires, 110 .

«Il est encore apparu dans un conseil, comme y présidant, et inspirant les résolutions qu'on y devait prendre pour la gloire de Dieu », Marie de l'Incarnation, 4: 230. 
$s^{\prime} y$ traictoit, ${ }^{53}$ eut la pensée que s'il estoit $\mathrm{S}^{\mathrm{t}}$ comme il le croyoit, il luy pourroit bien parler intellectuellement, et le pria interieurement de venir à luy. Incontinent. le pere daniel se leve, et le vient embrasser. Le P. N. le pria de luy dire ce que dieu demandoit plus particulierement $\grave{a}$ luy, ${ }^{54}$ à quoy il luy respondit ces paroles huronnes: SASANDIONRHENX NON8ARIH8ANDEAI, quj est la $5^{\mathbf{e}}$ demande du pater noster, pardonnez nous nos offenses, et embrasse le pere, luy imprimant en le baisant sur la joue une froideur sy grande qu'elle le fist esveiller et se trouva sy remply d'un sentiment de componction et d'apprehension de la justice de dieu particulierement sur sa pauvre Eglise que ce fut son occupation interieure plusieurs moys, et mesme jusques à la désolation du bourg et de sa Mission. ${ }^{55}$

Quelque temps après, le mesme pere vit en songe le pere Daniel deffunct ${ }^{56}$ dans une grande beauté, et le desir, voyant son bonheur et sa gloire, d'voir quelque chose de ses reliques pour l'honnorer davantage, luy fist faire une question: scavoir pourquoy et comment Dieu avoit permis qu'il eust esté tellement consumé des flammes, qu'il n'en estoit resté rien du tout. ${ }^{58}$ Il respondit que Dieu l'avoit bien recompensé, ayant faict profité beaucoup ces opprobes aux ames du purgatoire: ${ }^{59}$ ce quj imprima

53 «... à la vue de ce bienheureux tant de choses lui vinrent à l'esprit pour les lui demander, qu'il ne savait pas où commencer son entretien avec ce cher défunt », F. Martin, éd., Autobiographie du Père Chaumonot...., 84.

54 « Enfin lui-dit-il: «Apprenez-moi, mon Père, ce que ie dois faire pour être bien agréable à Dieu 》, ibid., 84 .

55 «Jamais», répondit le martyr, «ne perdez le souvenir de vos péchés ». C'est ce que le P.C. a observé depuis », ibid., 84-85.

56 « Ce saint martyr apparut peu de temps après sa mort à un Père de la Compagnie et de la Mission », Marie de l'Incarnation, 4: 229.

$57 \ll . .$. en estat de gloire, portant le visage d'un homme d'environ 30 ans, quoy qu'il soit mort à l'aage de quarante et huict », P. Ragueneau, Mémoires, 110.

58 «...que le corps de son serviteur fust traité si indignement apres sa mort, et tellement reduit en poudre, que mesme nous n'eussions pas le bonheur d'en pouvoir recueillir les cendres », P. Ragueneau, ibid., 110.

59 «MAGUS DOMINUS ET LAUDABILIS NIMIS, respondit-il, Ouy, dieu est grand et adorable à tout jamais. Il a jetté les yeux sur les opprobres de ce sien serviteur; et afin de les recompenser en dieu grand comme il est, il m'a donné quantité d'àmes quj estoient dans le purgatoire, lesquelles ont accompagné mon entrée et mon triomphe dans le Ciel », $P$. Ragueneau, ibid., 110. 
au cœur dud. pere une grande ferveur de devotion au soulagement de ces ames affligées, quj le porta à quelques actes heroiques en cette consideration, et particulierement d'humiliation et mortification interieures comme servant plus à ces ames que les oraisons ou austerités exterieures $\gg .^{60}$

De façon générale, nous pouvons dire que ces apparitions du Père Daniel ont été permises autant pour la gloire du saint que pour l'utilité des vivants. Aussi, grâce à ces dernières paroles venues de l'au-delà, est-il possible de découvrir d'autres aspects de son âme qui, jusqu'à présent, n'avaient pas été mis en lumière. Dans la première vision, le Père Chaumonot, désireux d'apprendre ce que Dieu attendait particulièrement de lui, reçut pour toute réponse cette parole du Pater: «Pardonnez-nous nos offenses ». C'était recommander à un apôtre une attitude essentielle, pleine d'un sain réalisme: le souvenir de ses fautes. Mais l'apôtre n'est pas responsable que de lui-même car les péchés des infidèles pèsent en quelque sorte sur ses épaules. Ainsi s'explique le devoir de la réparation qui lui est suggéré; peutêtre l'apôtre réalise-t-il par là plus de conversions que ne le pourraient faire ses plus éloquentes prédications. Par sa vie héroïque, le Père Daniel avait valu à de nombreux sauvages la grâce du Baptême; après sa mort, quel pouvait être son plus vif désir sinon de voir la même charité imitée par ses successeurs ?

Dans une seconde apparition, la sainteté du Père Daniel se manifeste avec l'éclat d'une figure rayonnante de jeunesse qui symbolise la pureté de son âme et le bonheur qu'elle ressent de connaître son Dieu face à face. Les paroles qu'il adressa en cette circonstance doivent retenir davantage notre attention: «MAGNUS DOMINUS ET LAUDABILIS NIMIS, respondit-il, Ouy, dieu est grand et adorable à tout jamais. Il a jetté les yeux sur les opprobres de ce sien serviteur; et afin de les recompenser en dieu grand comme il est, il m'a donné quantité d'ames quj estoient dans le purgatoire, lesquelles ont accompagné mon entrée et mon triomphe dans le Ciel ». ${ }^{61}$ Pour bien comprendre la portée d'une telle affirmation, il est bon de rappeler que cette apparition se

60 P. Joseph-A. Poncet, Mémoires, 111-112.

61 P. Ragueneau, Mémoires, 110. 
produisit quelques jours seulement après la mort du saint martyr; d'après Marie de l'Incarnation, ${ }^{62}$ son entrée au ciel suivit même immédiatement son martyre. Renonçons ici à toute comparaison qui ne pourrait être qu'inopportune, car les mérites des saints passent trop nos intelligences humaines pour qu'elles puissent tenter de les mesurer. Cette apparition prouve cependant d'une façon non équivoque la sainteté éminente du Père Daniel: non seulement elle lui vaut de passer directement de la terre au ciel, mais elle emporte encore dans son triomphe quantité d'âmes du purgatoire dont l'expiation volontaire du martyr avait abrégé le terrible supplice.

Ici s'achève notre entreprise. A l'aide de quelques documents, nous nous étions proposé de jeter un regard avide sur l'âme de saint Antoine Daniel pour essayer d'en percevoir les valeurs dominantes et surtout la splendide sainteté. Conscient de la témérité d'une telle enquête - difficulté du sujet, absence de documents personnels - nous ne pouvions, malgré tout, la négliger parce que nous aurions ainsi passé volontairement sous silence ses plus beaux titres de gloire: puissent-ils désormais attirer davantage notre admiration. Mais si la mauvaise interprétation de certains textes déforme ici ou là la personnalité du saint martyr, nous espérons du moins n'avoir pas faussé l'orientation essentielle de sa vie.

\section{CONCLUSION}

Après avoir exploré en détail la vie de saint Antoine Daniel ainsi que l'ascension constante de son âme, essayons maintenant de saisir, dans un seul regard, sa personnalité. Efforçons-nous de le comprendre comme l'ont pu faire ses meilleurs amis.

Il a été avant tout un homme d'une patience et d'une douceur admirables. D'après tous les témoignages, c'est là le trait dominant de son caractère. Ses manières réservées, mais pleines d'amabilité, charmaient les cœurs et inspiraient à tous un pro-

62 «... il Dieu m'a donné après ma mort un grand nombre d'âmes du purgatoire pour les emmener avec moi...», Marie de l'Incarnation, 4: 230. 
fond respect. Qualités naturelles, sans doute, mais surélevées à une dignité éminente par la grâce qui l'habitait totalement. Très affable par nature, le Père Daniel savait pourtant s'imposer avec vigueur et déployer une activité inlassable lorsque les exigences de l'apostolat le demandaient. Son zèle ardent pour la conversion des âmes dont toute sa vie fut un continuel exemple, il ne le puisait pas à d'autres sources que la charité.

L'Esprit-Saint était l'hôte habituel de son âme et lui communiquait surtout ce don de force «qui fait courir par mer et par terre les ouvriers de l'Évangile, et qui les fait des martyrs vivants avant que le fer et le feu les consume ${ }^{1}{ }^{1}$ On sait peu de choses, en somme, sur la vie spirituelle de saint Antoine Daniel, mais il paraît hors de doute qu'il ait été profondément engagé dans les voies mystiques. En cela, d'ailleurs, il est de son milieu et de son époque: nous connaissons suffisamment déjà de Brébeuf, Jogues, Garnier, qui tous furent ses compagnons d'apostolat; vers le même temps, vivaient à Québec Marie de l'Incarnation, Catherine de Saint-Augustin; le groupe des fondateurs de Montréal n'avait commencé son œuvre que six ans avant le martyre du Père Daniel. Dans l'histoire de la Nouvelle-France, cette période est toute remplie d'une ferveur mystique incomparable.

Saint Antoine Daniel nous apparaît donc comme un exemplaire splendide de l'apôtre engagé quotidiennement dans son ministère d'évangélisation. Il ne s'impose pas à nous par de brillantes qualités de chef ou d'organisateur; il a été plutôt celui qui réalise, celui qui rend tangible l'espérance d'autrui. Aussi, les six premières années de son séjour au Canada, malgré le travail réel qu'il accomplit pendant ce temps, ont-elles pour lui une valeur de purification surtout. Les changements d'emplois qu'on lui demande, presque chaque année, ne lui permettent pas d'envisager une occupation stable qui serait «sienne» à proprement parler; au contraire, ils l'obligent à reconnaître et à accepter qu'il sera avant tout un instrument à la disposition de ses supérieurs. C'est alors qu'il peut entreprendre cette longue étape de dix années remplies d'efforts soutenus, de résistances

1 Marie de l'Incarnation, 4: 255. 
à vaincre, de dangers continuels. Nul ne pourra dire exactement ce que furent ces longues années de labeur intense mais, considérant le terme de ces efforts, nous pouvons affirmer que le progrès spirituel du saint ne l'a cédé en rien à l'activité de l'apôtre. A l'heure de son martyre, il semble même que Dieu veuille le récompenser en lui accordant le privilège de toucher irrésistiblement les cœurs par la force de sa parole. En effet, ces conversions subites d'une foule de sauvages, autrefois arrogants et endurcis, paraissent bien être le fruit d'un charisme surnaturel.

Saint Antoine Daniel, nous semble-t-il, s'est montré durant toute sa vie le fidèle imitateur des grands apôtres de l'Église et de la Compagnie de Jésus. Une fois de plus, il a prouvé que devenir « in actione contemplativus » était une merveilleuse voie de sainteté.

Fernand Potvin, S.J. 